\title{
Estimation of Time-Limited Channel Spectra from Nonuniform Samples
}

\author{
J. Selva
}

\begin{abstract}
This paper deals with the estimation of a time-invariant channel spectrum from its own nonuniform samples, assuming there is a bound on the channel's delay spread. Except for this last assumption, this is the basic estimation problem in systems providing channel spectral samples. However, as shown in the paper, the delay spread bound leads us to view the spectrum as a band-limited signal, rather than the Fourier transform of a tapped delay line (TDL). Using this alternative model, a linear estimator is presented that approximately minimizes the expected root-mean-square (RMS) error for a deterministic channel. Its main advantage over the TDL is that it takes into account the spectrum's smoothness (time width), thus providing a performance improvement. The proposed estimator is compared numerically with the maximum likelihood (ML) estimator based on a TDL model in pilot-assisted channel estimation (PACE) for OFDM.
\end{abstract}

\section{INTRODUCTION}

The interpolation of band-limited signals from nonuniform samples is a recurrent topic in signal processing [1], [2]. Just to mention a few relevant scenarios, it appears in A/D conversion for wideband signals, where the existing fast A/D converters produce a nonuniform sampling scheme that must be corrected [3]-[9]. It also appears in image processing, where the sampling positions may be nonuniformly spaced due, for instance, to the geometry of the scenario in which a given image was captured [1. Ch. 6]. And, finally, the demodulation of an FM signal can be performed from its zero-crossings using nonuniform sampling techniques, [1, Ch. 16].

Copyright (c) 2015 IEEE. Personal use of this material is permitted. However, permission to use this material for any other purposes must be obtained from the IEEE by sending a request to pubs-permissions@ieee.org. The author is with the Dept. of Physics, Systems Engineering and Signal Theory (DFISTS), University of Alicante, P.O.Box 99, E-03080 Alicante, Spain (e-mail: jesus.selva@ua.es). This work was supported by the Spanish Ministry of Economy and Competitiveness (MINECO) under Project TEC2011-28201-C02-02. 
In the literature, there are two basic approaches for addressing the nonuniform sampling problem, that we may term "spectral" and "trigonometric". In the spectral approach, the signal's bandwidth is assumed known and the signal is modeled through either a nonuniform sampling series, or by passing to the frequency domain using the Fourier transform. This approach comprises classical results as Yen's [10] and recent results like [3], [11], and is the common way to model the problem in circuit design for $\mathrm{A} / \mathrm{D}$ conversion. In the trigonometric approach, the signal is approximated using a trigonometric polynomial, and there is no assumption about the signal's bandwidth. Then, the problem consists of efficiently estimating the polynomial coefficients and, afterward, the signal itself. Its theoretical foundation is the fact that the interpolation error converges to zero with the polynomial order, [12, Th. 1]. This approach has been extensively studied by Feichtinger et al. [13]-[18]. The usual zero-padding FFT interpolation [19, Sec. 3.11] is an instance of this approach.

One atypical scenario for nonuniform sampling is the estimation of a channel spectrum from its own nonuniform samples in OFDM [20]-[22]. In this scenario, the PACE (pilot-assisted channel estimation) techniques comprise the methods to perform this spectral estimation under various statistical assumptions. Among them, the maximum likelihood (ML) and the minimum mean-square (MMSE) estimators are benchmarks against which other estimators are compared in terms of statistical efficiency and complexity [23]. This scenario is atypical because the signal to be estimated is not a time- or spatial-domain signal, but the spectrum of a propagation channel that is sampled by means of pilot carriers. The techniques in PACE follow the trigonometric approach, and the polynomial's coefficients are usually referred to as TDL or channel impulse response (CIR) [24, Ch. 11].

This paper deals with the generic problem of estimating a time-limited static channel spectrum from its own nonuniform samples, assuming there is an upper bound on the channel's delay spread. In practice, such a bound can either be inferred from basic considerations about the channel (its type or geometry), or estimated, [25], [26]. We present a model in which the channel's spectrum is viewed as a band-limited signal and modeled using a sinc series. Then, we propose a linear estimator that approximately minimizes the RMS error. The problem addressed in this paper is the basic one in PACE for OFDM, but the inclusion of the delay spread bound as a new parameter produces a model following the spectral rather than the trigonometric approach. Since the proposed estimator makes use of additional information (delay spread bound), we may expect a performance improvement. The numerical examples confirm that this is so for the basic estimation problem in PACE for OFDM.

The paper has been organized as follows. In the next section, we first introduce the problem of estimating a static time-limited channel from its own nonuniform samples, and then follow the usual approach in which the channel's spectrum is approximated using a trigonometric polynomial, and then 
estimated by means of an ML estimator. We also discuss the usual justification of this trigonometric approach which is based on a so-called transmit-receive (tx-rx) pulse. Then, we state the spectral approach in Sec. III, and derive the estimator proposed in this paper in Sec. IV, termed spectral (SP) estimator. We discuss several aspects of this last estimator in Sec. $\mathrm{V}$ and, finally, compare the performances of the ML and SP estimators in Sec. VI numerically.

\section{A. Notation}

In the paper, we employ the following notation:

- New symbols or functions are introduced using “””.

- Signals are written in lower case letters and their corresponding spectra in upper case letters. Thus, $U(f)$ and $H(f)$ are the spectra of $u(t)$ and $h(t)$ respectively.

- Vectors and matrices are denoted in lower- and upper-case bold font, respectively, $(\boldsymbol{m}, \boldsymbol{M})$.

- $I$ stands for an identity matrix of proper size.

- For a given matrix $\boldsymbol{A}$ or vector $\boldsymbol{a},[\boldsymbol{A}]_{p, q}$ and $[\boldsymbol{a}]_{r}$ respectively denote the $p, q$ component of $\boldsymbol{A}$, and the $r$ th component of $\boldsymbol{a}$.

- $\boldsymbol{A}^{H}$ and $\boldsymbol{A}^{T}$ respectively denote the Hermitian and transpose of $\boldsymbol{A}$.

- $\boldsymbol{A}^{\dagger}$ denotes the pseudo-inverse of $\boldsymbol{A}$.

- $\boldsymbol{a} \odot \boldsymbol{b}$ stands for the component-wise product of vectors $\boldsymbol{a}$ and $\boldsymbol{b}$, that is, $[\boldsymbol{a} \odot \boldsymbol{b}]_{m}=[\boldsymbol{a}]_{m}[\boldsymbol{b}]_{m}$.

- $\mathbb{E}\{\cdot\}$ denotes the expectation operator.

\section{ESTIMATION OF A TIME-LIMITED CHANNEL SPECTRUM FROM ITS OWN NONUNIFORM SAMPLES}

In a variety of applications, the spectrum of a propagation channel must be estimated from a set of noisy samples. For a simple static channel, we may describe this situation by considering a set of frequencies $f_{m}, f_{m}<f_{m+1}, m=1, \ldots, M$, and a set of samples

$$
V_{m} \equiv H\left(f_{m}\right)+E_{m}
$$

where $H(f)$ is the channel's spectrum, and $E_{m}$ are zero-mean independent complex Gaussian noise samples of equal variance $\sigma_{E}^{2}$. The objective is then to design an estimator $\hat{H}(f ; \boldsymbol{v})$ of $H(f)$, where

$$
[\boldsymbol{v}]_{m+1} \equiv V_{m}, m=1, \ldots, M
$$

and a proper error measure for this design is the expected quadratical error

$$
\mathbb{E}\left\{|H(f)-\hat{H}(f ; \boldsymbol{v})|^{2}\right\}
$$

where we view $H(f)$ as deterministic for simplicity. 
Eqs. (1) and (2) describe a basic estimation problem in systems providing channel spectral samples, like OFDM systems equipped with pilot carriers [20]-[22], and channel sounding systems in general. In some applications, the problem is more complex than the one just stated, given that the channel's response is assumed time variant. However, in this last case the usual models are extensions of (1) that take into account the Doppler shifts, [21].

The trigonometric approach is the usual way to address the estimation of $H(f)$ from (1), and is based on the interpolator

$$
H(f) \approx \sum_{n=n_{1}}^{n_{2}} h_{n} e^{-j 2 \pi n T f},
$$

for specific truncation indices $n_{1}$ and $n_{2}$, time period $T>0$, and set of coefficients $h_{n}$, [24, Ch. 11]. This interpolator greatly simplifies the estimation problem, because after inserting (4) into (1), we obtain the model

$$
V_{m} \approx \sum_{n=n_{1}}^{n_{2}} h_{n} e^{-j 2 \pi n T f_{m}}+E_{m},
$$

in which the only parameters to estimate are the coefficients $h_{n}, n_{1} \leq n \leq n_{2}$. At this point, there exists a variety of estimators for $h_{n}$ and, in turn, $H(f)$. One of the reference estimators is that based on the ML principle [23], in which the estimate of $h_{n}, \hat{h}_{\mathrm{ML}, n}$, is the least-squares solution of the linear system

$$
V_{m} \approx \sum_{n=n_{1}}^{n_{2}} \hat{h}_{\mathrm{ML}, n} e^{-j 2 \pi n T f_{m}} .
$$

From $\hat{h}_{\mathrm{ML}, n}$, the ML estimate of $H(f)$ is the result of replacing $h_{n}$ with $\hat{h}_{\mathrm{ML}, n}$ in $(4)$. This last estimate can be concisely written as

$$
\hat{H}_{\mathrm{ML}}(f ; \boldsymbol{v}) \equiv \phi(f)^{T} \boldsymbol{\Phi}^{\dagger} \boldsymbol{v}
$$

where

$$
\begin{gathered}
{[\boldsymbol{\phi}(f)]_{n-n_{1}+1} \equiv e^{-j 2 \pi n T f},} \\
{[\boldsymbol{\Phi}]_{m, n-n_{1}+1} \equiv e^{-j 2 \pi n T f_{m}},} \\
\left(m=1,2, \ldots, M, n=n_{1}, n_{1}+1, \ldots, n_{2}\right) .
\end{gathered}
$$

The usual justification for the trigonometric interpolator in (4) is based on an analytical tool, the socalled (tx-rx) pulse, [24, Ch. 11]. A tx-rx pulse $u(t)$ is a band-limited signal whose spectrum $U(f)$ selects the band in which the estimation is to be performed. More precisely, for a fixed initial frequency $f_{u o}$ and a sampling period $T>0$, with $f_{u o} \leq f_{1}$ and $f_{M} \leq f_{u o}+1 / T$, the spectrum $U(f)$ of such pulse fulfills the conditions,

1) $U(f)=1$ if $f_{1} \leq f \leq f_{M}$. 
2) $U(f)=0$ if $f \leq f_{u o}$ or $f_{u o}+1 / T \leq f$, [band-limited $\left.u(t)\right]$.

3) $u(t)$ has finite energy.

Typical pulses $u(t)$ are a modulated sinc or raised cosine, though $u(t)$ is not even mentioned in most references in the literature.

For justifying (4) using $u(t)$, note that this pulse allows us to state the problem in (1) and $(3)$ in terms of the spectrum

$$
H_{u}(f) \equiv H(f) U(f)
$$

rather than $H(f)$, given that $U(f)$ neither affects the value of $H(f)$ at the sampling frequencies $f_{m}$, $m=1,2 \ldots, M$, nor at the possible estimation frequencies $f$. But now $H_{u}(f)$ can be modeled as the discrete-time Fourier transform (DTFT) of the sequence $h_{u, n} \equiv T(h * u)(n T), n \in \mathbb{Z}$, [27, Sec. 4.26]. Specifically, we have

$$
H_{u}(f)=\sum_{n=-\infty}^{\infty} h_{u, n} e^{-j 2 \pi n T f} .
$$

Next, the truncation of this DTFT at two indices $n_{1}$ and $n_{2}$ produces a trigonometric polynomial,

$$
H_{u}(f) \approx \sum_{n=n_{1}}^{n_{2}} h_{u, n} e^{-j 2 \pi n T f} .
$$

This polynomial also approximates $H(f)$ in the band in which $U(f)=1$ and, therefore, we have just validated the interpolator in (4) if we identify $h_{u, n}$ with $h_{n}$.

In this trigonometric approach, the truncation at indices $n_{1}$ and $n_{2}$ constitutes a way to restrict the smoothness of $H(f)$, given that in performing the truncation we are regarding the harmonics $h_{u, n} e^{-j 2 \pi n T f}$ for $n<n_{1}$ or $n>n_{2}$ as negligible. In practice, however, we may often give a stronger description of this smoothness, given that we may find out an upper bound $T_{h o}$ on the delay spread of $h(t)$ such that $h(t)=0$ if $t<0$ or $t>T_{h o}$. This is so for the following reasons:

- In practice, we may determine an upper bound on the channel's delay spread $T_{h o}$ from basic considerations about the channel, (channel's average geometry, type of channel, etc) [25], or by estimating it [26].

- The synchronization circuits in practical receivers deliver a reference that may be used to shift the time variable in $h(t)$, so that the support of $h(t)$ lies in $\left[0, T_{h o}\right]$.

With the range $\left[0, T_{h o}\right]$ bounding the support of $h(t)$, we have that $H(f)$ can be viewed as a bandlimited signal whose spectrum lies in $\left[-T_{h o}, 0\right]$. This is a direct consequence of the duality property of the Fourier transform, given that the spectrum of $H(f)$ is $h(-t)$. So, we have that the estimation problem can be tackled using sampling theory tools. We follow this approach, termed spectral, in the next section. 


\section{STATEMENT OF THE SPECTRAL APPROACH}

As already discussed, the tx-rx pulse is the key for obtaining a proper interpolation model for $H(f)$ in the trigonometric approach, given that it enables the use of the DTFT in 6. Note, however, that this pulse plays no role in the final interpolator (4). In the spectral approach, we proceed to introduce another pulse, denoted $w(t)$, and also consider its convolution with $h(t)$. Thus, we define the following response and spectrum

$$
h_{w}(t) \equiv(h * w)(t), \quad H_{w}(f) \equiv H(f) W(f) .
$$

However, the conditions we impose on $w(t)$ are different. For a time width $T_{w}>0$, they are the following,

1) $W(f) \approx e^{-j \pi T_{w} f}$ if $f_{1} \leq f \leq f_{M}$.

2) $w(t)=0$ if $t$ is outside the range $\left[0, T_{w}\right]$, [time-limited $\left.w(t)\right]$.

3) $w(t)$ has finite energy $\mathcal{E}_{w}$.

The first condition will have the same function as the corresponding condition on $U(f)$ in the previous section, but here it is an approximation due to condition 2). This last condition makes it possible to exploit the knowledge of $T_{h o}$, given that now $h_{w}(t)$ has a known duration

$$
T_{h} \equiv T_{h o}+T_{w}
$$

Finally, the third condition will allow us to employ basic tools like the Cauchy-Schwarz inequality in the sequel. A pulse $w(t)$ fulfilling these three conditions can be constructed by multiplying a proper window function with a sinc pulse (App. A though, as in the trigonometric approach, it is not necessary to specify this pulse in order to derive an estimator.

Next, consider $h_{w}(t)$. This response has finite energy, denoted $\mathcal{E}_{H, w}$, for the usual responses of the form

$$
h(t)=h_{d}(t)+\sum_{k=1}^{K} a_{k} \delta\left(t-\tau_{k}\right),
$$

for a finite number of deltas $K$ and a diffuse component $h_{d}(t)$ with finite $L^{1}$ norm, i.e, following

$$
\int_{0}^{T_{h o}}\left|h_{d}(t)\right| d t<\infty
$$

So, its Fourier transform $H_{w}(f)$ can be viewed as a band-limited signal with spectrum lying in $\left[-T_{h}, 0\right]$, and it can be represented using a sinc series. In order to introduce this series, it is convenient to re-state the estimation problem in terms of the following normalized spectrum,

$$
\eta(x) \equiv H_{w}\left(\frac{x}{T_{h}}\right) e^{j \pi x},
$$


where $x$ denotes a new variable. It can be easily checked that the spectrum of $\eta(x)$ lies in $[-1 / 2,1 / 2]$ and its energy is

$$
\mathcal{E}_{\eta} \equiv T_{h} \mathcal{E}_{H, w}
$$

Now, the Shannon sampling theorem yields the well-known sinc series representation for $\eta(x)$,

$$
\eta(x)=\sum_{p=-\infty}^{\infty} \eta(p) \operatorname{sinc}(x-p) .
$$

Note that if $f_{1} \leq x / T_{h} \leq f_{M}$, then $\eta(x)$ is also a normalized version of $H(f)$ due to condition 1$)$ on $W(f)$,

$$
\begin{aligned}
\eta(x) & =H\left(\frac{x}{T_{h}}\right) W\left(\frac{x}{T_{h}}\right) e^{j \pi x} \approx H\left(\frac{x}{T_{h}}\right) e^{-j \pi T_{w} x / T_{h}} e^{j \pi x} \\
& =H\left(\frac{x}{T_{h}}\right) e^{j \pi\left(1-T_{w} / T_{h}\right) x} .
\end{aligned}
$$

In terms of $\eta(x)$, the estimation problem in (1) can be re-stated as

$$
z_{m}=\eta\left(x_{m}\right)+\epsilon_{m}
$$

where $z_{m}, x_{m}$ and $\epsilon_{m}$ are related with $V_{m}, f_{m}$ and $E_{m}$ through (9). Specifically, we have the definitions

$$
\begin{aligned}
& \text { Sampling abscissas: } x_{m} \equiv f_{m} T_{h} \\
& \text { Data samples: } z_{m} \equiv V_{m} e^{j \pi\left(1-T_{w} / T_{h}\right) x_{m}} \\
& \text { Noise samples: } \epsilon_{m} \equiv E_{m} e^{j \pi\left(1-T_{w} / T_{h}\right) x_{m}}
\end{aligned}
$$

Additionally, (3) is equivalent to designing an estimator $\hat{\eta}(f ; \boldsymbol{z})$ of the deterministic signal $\eta(x)$ with small error given by

$$
\mathbb{E}\left\{|\eta(x)-\hat{\eta}(x ; \boldsymbol{z})|^{2}\right\},
$$

where

$$
[\boldsymbol{z}]_{m+1} \equiv z_{m}, m=1,2, \ldots, M
$$

Given an estimator $\hat{\eta}(x ; \boldsymbol{z})$ of $\eta(x)$ for $f_{1} \leq x / T_{h} \leq f_{M}$, the corresponding estimator of $H(f)$ can be easily obtained by inverting (9),

$$
\begin{aligned}
\hat{H}(f ; \boldsymbol{v}) & =\hat{\eta}\left(f T_{h} ; \boldsymbol{z}\right) e^{-j \pi\left(1-T_{w} / T_{h}\right) f T_{h}} \\
& =\hat{\eta}\left(f T_{h} ; \boldsymbol{z}\right) e^{-j \pi T_{h o} f}
\end{aligned}
$$


In the spectral approach, we propose to find the linear estimator of $\eta(x)$ that minimizes the maximum of (11) over the set of spectra $\eta(x)$ with energy at most $\mathcal{E}_{\eta}$. Specifically, the estimator will be termed "spectral" (SP) in the sequel and has the form

$$
\hat{\eta}_{\mathrm{SP}}(x ; \boldsymbol{z}) \equiv \sum_{m=1}^{M} z_{m} \hat{c}_{\mathrm{SP}, m}(x),
$$

where the coefficients $\hat{c}_{\mathrm{SP}, m}(x)$ are given by the minimax problem

$$
\left\{\hat{c}_{\mathrm{SP}, m}(x)\right\}=\arg \min _{\left\{c_{m}(x)\right\}} \max _{\eta(x)} \mathbb{E}\left\{\left|\eta(x)-\sum_{m=1}^{M} z_{m} c_{m}(x)\right|^{2}\right\} .
$$

In this last expression, the curly braces $\{\cdot\}$ denote the corresponding set of coefficients for $m=$ $1,2, \ldots, M$, and the inner maximum is taken over the set of possible $\eta(x)$ with energy at most $\mathcal{E}_{\eta}$ and spectrum in $[-1 / 2,1 / 2]$. The linearity constraint in $[13$ is convenient for two reasons. First, as is well known the derivation of an estimator is greatly simplified under this constraint. And second, the optimal interpolator in the noise-free setting is linear for bounded band-limited signals [28]. This last argument implies that for high signal-to-noise (SNR) ratios the linearity constraint is not a significant limitation.

We derive a closed-form expression for $\hat{\eta}_{\mathrm{SP}}(x ; \boldsymbol{z})$ in the next section.

\section{CLOSED-FORM EXPRESSION OF THE PROPOSED ESTIMATOR}

Let us derive an explicit expression for $\hat{\eta}_{\mathrm{SP}}(x ; \boldsymbol{z})$. We first apply the condition $\mathbb{E}\left\{\epsilon_{m}\right\}=0$ to the cost function in this problem to separate the mismatch and noise terms:

$$
\begin{aligned}
\mathbb{E}\left\{\left|\eta(x)-\sum_{m=1}^{M} z_{m} c_{m}(x)\right|^{2}\right\} & \\
=\mathbb{E}\left\{\left|\eta(x)-\sum_{m=1}^{M}\left(\eta\left(x_{m}\right)+\epsilon_{m}\right) c_{m}(x)\right|^{2}\right\} & \\
& =\left|\eta(x)-\sum_{m=1}^{M} \eta\left(x_{m}\right) c_{m}(x)\right|^{2}+\sigma_{E}^{2} \sum_{m=1}^{M}\left|c_{m}(x)\right|^{2} .
\end{aligned}
$$

At this point, we insert the sinc series (8) into this expression, and then use the Cauchy-Schwarz inequality to obtain an upper bound for fixed $c_{m}(x)$. We only need to consider the first term:

$$
\begin{aligned}
\left|\eta(x)-\sum_{m=1}^{M} \eta\left(x_{m}\right) c_{m}(x)\right|^{2}=\mid \sum_{p=-\infty}^{\infty} \eta(p) \operatorname{sinc}(x-p) & \\
& -\left.\sum_{m=1}^{M} \sum_{p=-\infty}^{\infty} \eta(p) \operatorname{sinc}\left(x_{m}-p\right) c_{m}(x)\right|^{2}
\end{aligned}
$$




$$
\begin{aligned}
=\mid \sum_{p=-\infty}^{\infty} \eta(p)(\operatorname{sinc}( & \left.x-p)-\sum_{m=1}^{M} \operatorname{sinc}\left(x_{m}-p\right) c_{m}(x)\right)\left.\right|^{2} \\
& \leq \mathcal{E}_{\eta} \sum_{p=-\infty}^{\infty}\left|\operatorname{sinc}(x-p)-\sum_{m=1}^{M} \operatorname{sinc}\left(x_{m}-p\right) c_{m}(x)\right|^{2} .
\end{aligned}
$$

Besides this bound is tight; (for this point see Sec. V-A).

Substituting into (15), we obtain

$$
\begin{aligned}
\mathbb{E}\left\{\left|\eta(x)-\sum_{m=1}^{M} z_{m} c_{m}(x)\right|^{2}\right\} & \\
\mathcal{E}_{\eta} \sum_{p=-\infty}^{\infty}\left|\operatorname{sinc}(x-p)-\sum_{m=1}^{M} \operatorname{sinc}\left(x_{m}-p\right) c_{m}(x)\right|^{2} & \\
& +\sigma_{E}^{2} \sum_{m=1}^{M}\left|c_{m}(x)\right|^{2} .
\end{aligned}
$$

Next, we minimize this bound in the set of coefficients $c_{m}(x)$. The minimum occurs for real coefficients $c_{m}(x)$, given that $\operatorname{sinc}(x)$ is a real function whenever $x$ is real. So, for real $c_{m}(x)$ we expand the argument of the first sum in 17 to obtain

$$
\begin{aligned}
&\left|\operatorname{sinc}(x-p)-\sum_{m=1}^{M} \operatorname{sinc}\left(x_{m}-p\right) c_{m}(x)\right|^{2} \\
&=\operatorname{sinc}^{2}(x-p)-2 \sum_{m=1}^{M} \operatorname{sinc}(x-p) \operatorname{sinc}\left(x_{m}-p\right) c_{m}(x) \\
&+\sum_{m=1}^{M} \sum_{m^{\prime}=1}^{M} \operatorname{sinc}\left(x_{m}-p\right) \operatorname{sinc}\left(x_{m^{\prime}}-p\right) c_{m}(x) c_{m^{\prime}}(x) .
\end{aligned}
$$

In this expression, note that all terms contain a product of two sinc functions. But the sinc function has the property

$$
\sum_{p=-\infty}^{\infty} \operatorname{sinc}(y-p) \operatorname{sinc}\left(y^{\prime}-p\right)=\operatorname{sinc}\left(y-y^{\prime}\right)
$$

which is valid for any $y$ and $y^{\prime}$. So summing 18 for $p \in \mathbb{Z}$ and using this last property, we obtain

$$
\begin{aligned}
& \sum_{p=-\infty}^{\infty}\left|\operatorname{sinc}(x-p)-\sum_{m=1}^{M} \operatorname{sinc}\left(x_{m}-p\right) c_{m}(x)\right|^{2} \\
= & 1-2 \sum_{m=1}^{M} \operatorname{sinc}\left(x-x_{m}\right) c_{m}(x)
\end{aligned}
$$$$
+\sum_{m=1}^{M} \sum_{m^{\prime}=1}^{M} \operatorname{sinc}\left(x_{m}-x_{m^{\prime}}\right) c_{m}(x) c_{m^{\prime}}(x) .
$$ 
Next, substitute this formula into (17),

$$
\begin{aligned}
& \mathbb{E}\left\{\left|\eta(x)-\sum_{m=1}^{M} z_{m} c_{m}(x)\right|^{2}\right\} \\
& \leq \mathcal{E}_{\eta}-2 \mathcal{E}_{\eta} \sum_{m=1}^{M} \operatorname{sinc}\left(x-x_{m}\right) c_{m}(x) \\
& \\
& \quad+\mathcal{E}_{\eta} \sum_{m=1}^{M} \sum_{m^{\prime}=1}^{M} \operatorname{sinc}\left(x_{m}-x_{m^{\prime}}\right) c_{m}(x) c_{m^{\prime}}(x)
\end{aligned}
$$$$
+\sigma_{E}^{2} \sum_{m=1}^{M}\left|c_{m}(x)\right|^{2}
$$

This inequality can be concisely written as

$$
\mathbb{E}\left\{\left|\eta(x)-\sum_{m=1}^{M} z_{m} c_{m}(x)\right|^{2}\right\} \leq
$$

$$
\mathcal{E}_{\eta}\left(\boldsymbol{c}(x)^{T}(\boldsymbol{G}+\boldsymbol{I} / \gamma) \boldsymbol{c}(x)-2 \boldsymbol{c}(x)^{T} \boldsymbol{g}(x)+1\right),
$$

where

$$
\begin{aligned}
& \gamma \equiv \frac{\mathcal{E}_{\eta}}{\sigma_{E}^{2}},[\boldsymbol{G}]_{m, m^{\prime}} \equiv \operatorname{sinc}\left(x_{m}-x_{m^{\prime}}\right), \\
& {[\boldsymbol{g}(x)]_{m} } \equiv \operatorname{sinc}\left(x-x_{m}\right),[\boldsymbol{c}(x)]_{m} \equiv c_{m}(x), \\
& 1 \leq m \leq M, 1 \leq m^{\prime} \leq M .
\end{aligned}
$$

Note that the bound in 19$]$ is a quadratic form in $\boldsymbol{c}_{m}(x)$ whose matrix $\boldsymbol{G}+\boldsymbol{I} / \gamma$ is positive definite. This fact implies that it has a unique global minimum which is attained at

$$
\hat{\boldsymbol{c}}(x) \equiv(\boldsymbol{G}+\boldsymbol{I} / \gamma)^{-1} \boldsymbol{g}(x)
$$

The corresponding minimum value is obtained by substituting this last expression into (19)

$$
\begin{aligned}
\mathbb{E}\left\{\left|\eta(x)-\sum_{m=1}^{M} z_{m} c_{m}(x)\right|^{2}\right\} & \\
& \leq \mathcal{E}_{\eta}\left(1-\boldsymbol{g}(x)^{T}(\boldsymbol{G}+\boldsymbol{I} / \gamma)^{-1} \boldsymbol{g}(x)\right) .
\end{aligned}
$$

In summary, the solution of the minimax problem in (14) is

$$
\hat{\eta}_{\mathrm{SP}}(x ; \boldsymbol{z})=\boldsymbol{g}(x)^{T}(\boldsymbol{G}+\boldsymbol{I} / \gamma)^{-1} \boldsymbol{z} .
$$

The estimator for $H(f)$ is readily obtained from 12 ,

$$
\hat{H}_{\mathrm{SP}}(f ; \boldsymbol{v})=e^{j \pi T_{h o} f} \boldsymbol{g}\left(T_{h} f\right)^{T}(\boldsymbol{G}+\boldsymbol{I} / \gamma)^{-1}(\boldsymbol{v} \odot \boldsymbol{\psi}),
$$


where

$$
[\boldsymbol{\psi}]_{m} \equiv e^{j \pi T_{h} f_{m}}, \quad[\boldsymbol{v}]_{m} \equiv V_{m}
$$

Additionally, the bound in 20 as a function of the frequency $f$ is

$$
\mathcal{E}_{\eta}\left(1-\boldsymbol{g}\left(T_{h} f\right)^{T}(\boldsymbol{G}+\boldsymbol{I} / \gamma)^{-1} \boldsymbol{g}\left(T_{h} f\right)\right) .
$$

It is worth mentioning that 21$]$ coincides with the minimum energy interpolator in [10] when $\gamma \rightarrow \infty$.

\section{COMments}

\section{A. Sharpness of the SP estimator}

In the derivation of the SP estimator, the only relaxation has been the Cauchy-Schwarz inequality is (16). However, as is well known, this inequality is attained by the sequence

$$
\eta(p)=K\left(\operatorname{sinc}(x-p)-\sum_{m=1}^{M} \operatorname{sinc}\left(x_{m}-p\right) c_{m}^{*}(x)\right), \quad p \in \mathbb{Z},
$$

(matched-filter principle), where $K$ is selected to set the energy equal to $\mathcal{E}_{\eta}$. Besides, this sequence corresponds to the signal

$$
\eta(y)=K\left(\operatorname{sinc}(x-y)-\sum_{m=1}^{M} \operatorname{sinc}\left(x_{m}-y\right) c_{m}^{*}(x)\right),
$$

whose energy is $\mathcal{E}_{\eta}$ and whose spectrum lies in $[-1 / 2,1 / 2]$. So the estimator is optimal under the following conditions:

1) We restrict the estimator to be linear.

2) The error in the approximation $W(f) \approx e^{-j \pi T_{w} f}, f_{1} \leq f \leq f_{M}$ is negligible.

3) The set of possible spectra $\eta(x)$ is the set of complex finite-energy band-limited functions with spectrum in $[-1 / 2,1 / 2]$ and energy at most $\mathcal{E}_{\eta}$.

\section{B. Estimate of $\gamma$ parameter}

The SP estimator has been designed for a deterministic spectrum $H(f)$ and requires the parameter $\gamma$. In practice, $H(f)$ is usually random and the value of $\gamma$ unknown. So, in order to make the SP usable for random $H(f)$, we propose to substitute $\gamma$ with the following estimate

$$
\hat{\gamma} \equiv \frac{\hat{P}_{H}}{\sigma_{E}^{2}}
$$

where $\hat{P}_{H}$ is an estimate of the average spectral power, given by

$$
P_{H} \equiv \frac{1}{f_{M}-f_{1}} \int_{f_{1}}^{f_{M}} \mathbb{E}\left\{|H(f)|^{2}\right\} d f .
$$

This is the estimate of $\gamma$ that will be employed in the numerical examples in Sec. VI 


\section{Selection of $T_{h} / T_{h o}$ ratio}

The proposed estimator depends on a pulse $w(t)$ of time-width $T_{w}$ which is not specified. Therefore, if $T_{h}$ is fixed then it is not clear for what $T_{h o}$ the estimator is usable, given that $T_{h}=T_{h o}+T_{w}$. The answer is that it is usable for any $T_{h o}$ following $T_{h o}<T_{h}$. This is so for the following two reasons. First, we have that for any $T_{w}>0$, we may obtain any product $B_{w} T_{w}$, simply by selecting a proper $B_{w}$. And second, there exist pulses as the one in App. A for which the conditions on $w(t)$ hold for a sufficiently large $B_{w} T_{w}$ product. In practice, if $T_{h o} \approx T_{h}$ or, equivalently, if $T_{h} / T_{h o} \approx 1$ then the estimator has a poor performance for single-delay channels with delay either close to zero or $T_{h o}$. This aspect is analyzed in Sec. VI-B numerically.

\section{Computational burden}

The computational burden of the proposed method can be easily derived from (21). We have the following operations and complexity orders:

- Computation of matrix $\boldsymbol{G}+\boldsymbol{I} / \gamma: O\left(M^{2}\right)$.

- Inversion of matrix $\boldsymbol{G}+\boldsymbol{I} / \gamma: O\left(M^{3}\right)$.

- Multiplication of $(\boldsymbol{G}+\boldsymbol{I} / \gamma)^{-1}$ and $\boldsymbol{z}: O(M)$.

- Multiplication of $\boldsymbol{g}(x)$ and $(\boldsymbol{G}+\boldsymbol{I} / \gamma)^{-1} \boldsymbol{z}$ assuming $N$ estimation frequencies: $O(M N)$.

The most expensive operation computationally is the inversion in step 2). Note, however, that such inversion is also involved in the ML estimator in (5), in which it is necessary to compute the pseudoinverse. If the set of frequencies $f_{m}$ is constant, then steps 1) and 2) can be pre-computed and the corresponding operations spared. In this last case, the total cost is linear in both the number of data and estimation frequencies, $[O(M N)$ complexity].

\section{NuMERICAL EXAMPLE}

In order to assess the SP estimator, we proceed to compare it with the ML estimator in (5). We present the simulation setup in the next subsection, and then assess the performance of both estimators for single delay channels in Sec. VI-B, and a standard channel model in Sec. VI-C.

\section{A. Numerical example setup}

1) OFDM signal: Following the example in [23, Sec. IV.C], we select an OFDM signal with the following parameters:

- DFT size: 512. 
- Number of modulated carriers: 433.

- Number of pilots: $M=28$.

- Indices of pilot carriers: $i_{m}=40+16 m, m=0,1, \ldots, M-1$.

- Carrier spacing: $\Delta f \equiv 1 /(N T)$. This parameter and in turn $T$ will be specified as a function of the spectral oversampling ratio, defined in the sequel.

2) Selection of noise variance $\sigma_{E}^{2}$ : We have defined the signal to noise ratio

$$
\mathrm{SNR} \equiv \frac{P_{H}}{\sigma_{E}^{2}}
$$

where $P_{H}$ was defined in $(23)$ and depends on the channel model. Then we have set $\mathrm{SNR}=30 \mathrm{~dB}$ and $\sigma_{E}^{2}=\mathrm{SNR} \cdot P_{H}$.

3) Estimate of $\gamma$ in the proposed estimator: We have used the estimate in Sec. V-B.

4) Carrier spacing: We select $\Delta f$ as a function of the oversampling factor. Specifically, the oversampling ratio is defined by

$$
\alpha \equiv \frac{1}{B_{a v} T_{h}}
$$

where $B_{a v}$ is the average pilot spacing

$$
B_{a v} \equiv \frac{i_{M-1}-i_{0}}{M-1} \Delta f
$$

So, for a specific $\alpha$, we take

$$
\Delta f=\frac{M-1}{\alpha T_{h}\left(i_{M-1}-i_{0}\right)} .
$$

5) Estimators: We compare in the next sub-sections three estimators:

- ML: Trigonometric ML estimator in (5) with the number of taps providing the smallest RMS error.

- SP: Proposed spectral estimator in (22) with $T_{h}=T_{h o}$.

- SP60: This last estimator but with $\gamma=60 \mathrm{~dB}$. For higher values of this parameter, the matrix inverted in (22) becomes ill conditioned. Therefore this estimator can be viewed as the one assuming "infinite" $\gamma$

\section{B. Assessment for single-delay channels}

Fig. 1 shows the difference in RMS error between the ML and SP estimators, for each possible delay and frequency, and three oversampling factors, $\alpha=2,4$, and 8. For these factors, the number of taps in the ML estimator were 17, 13, and 13 respectively. For $\alpha=2$, we can see that the estimators have similar performance, and the SP estimator performs better away from either the limit frequencies or delays. For $\alpha=4,8$, the SP estimator performs worse at the limits of the delay range, i.e, close to delays 0 or $T_{h}$. 


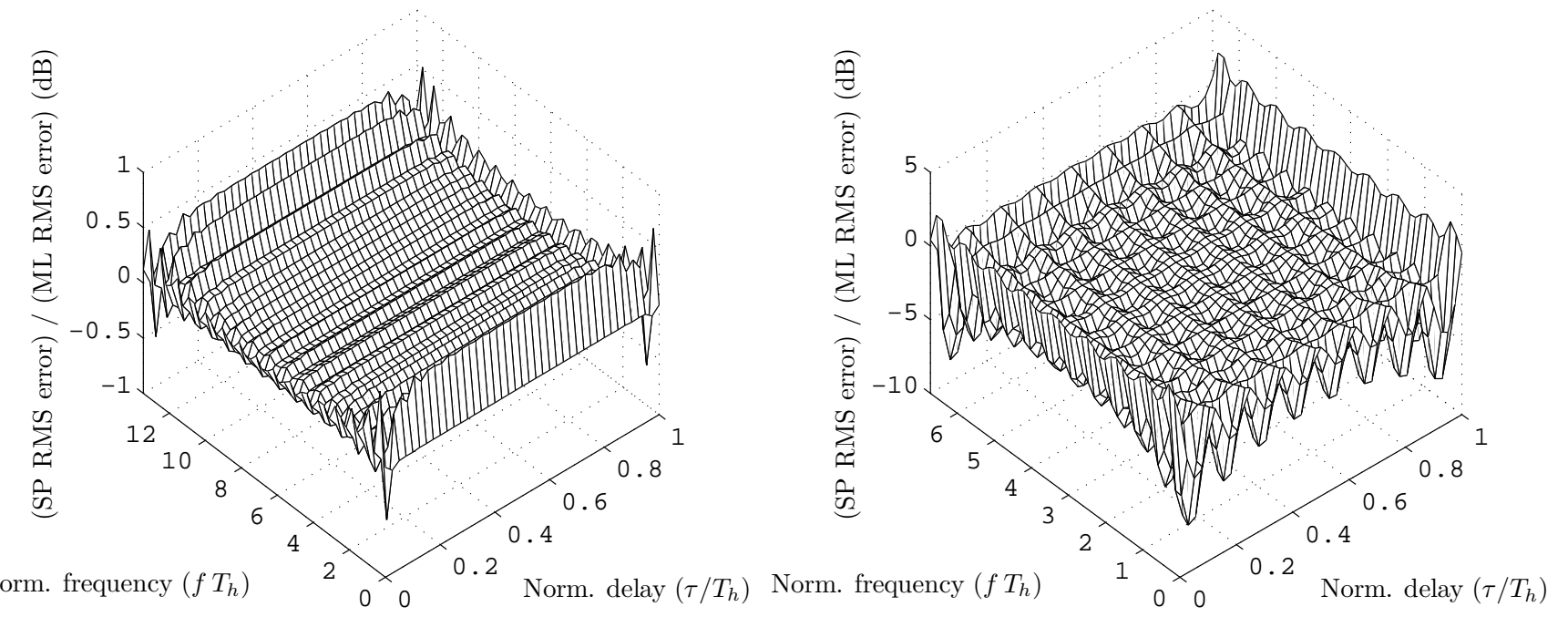

(a) $\alpha=2$, (ML with 17 taps)

(b) $\alpha=4$, (ML with 13 taps)

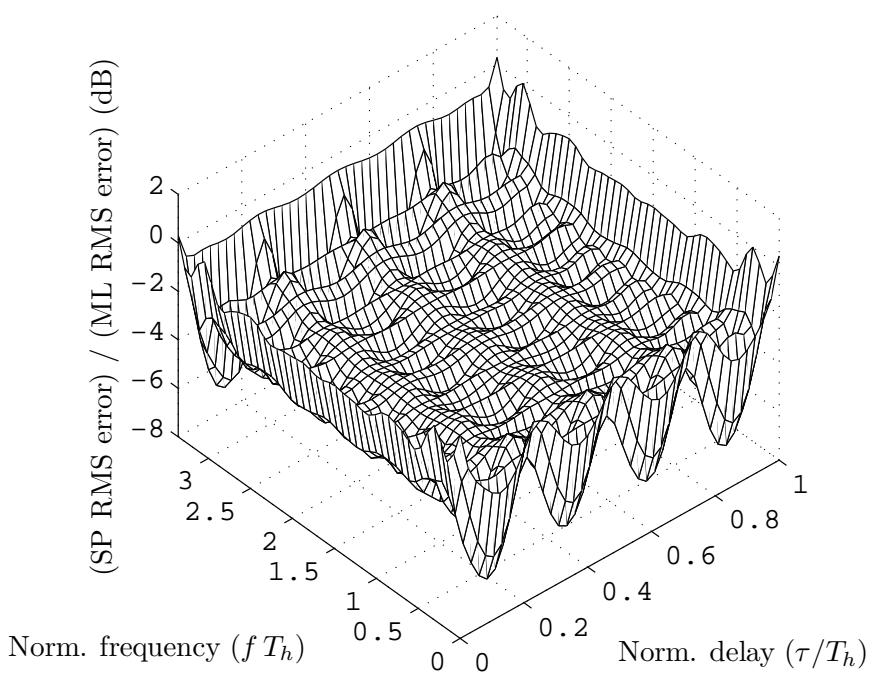

(c) $\alpha=8$, (ML with 13 taps)

Fig. 1. Difference in dBs between the RMS errors of the SP and ML estimators for oversampling factors $\alpha=2,4$, and 8 .

However, these values can be easily excluded by selecting a $T_{h} / T_{h o}$ ratio slightly above 1 . Except in this last case, the SP estimator outperforms the ML estimator. In average, the improvement of the SP over the ML estimator is $-0.1 \mathrm{~dB}, 1.76 \mathrm{~dB}$, and $3.5 \mathrm{~dB}$ for $\alpha=2,4$, and 8 respectively.

Fig. 2 shows the performance of the SP estimator for $\alpha=0.25$. Note that the error increases strongly close to either the zero or $T_{h}$ delay. This increase can be eliminated by selecting $T_{h o}$ slightly smaller than $T_{h}$. 


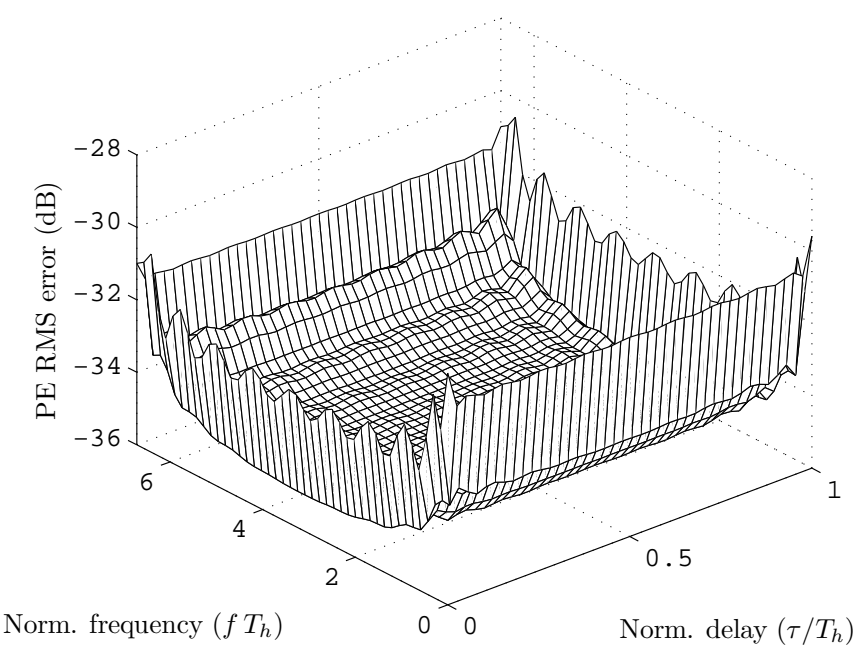

Fig. 2. RMS error of proposed estimator.

\section{Assessment for a standard channel model}

We select the indoor propagation model in the ITU-R M.1225 recommendation (channel A, no Doppler), [29. Table 3, p. 28], but assume the delays to lie inside the range $\left[0, T_{h o}\right]$ with $T_{h o}=340$ nsec. For this, we assume the first delay is at $t=20 \mathrm{nsec}$. In this model, the channel response realizations take the form

$$
h(t)=\sum_{k=1}^{6} a_{k} \delta\left(t-\tau_{k}\right)
$$

where the amplitudes $a_{k}$ are independent and follow a complex Gaussian zero-mean distribution. The delays $\tau_{k}$ and the variances of the amplitudes $a_{k}$ are the following:

\begin{tabular}{l|c|c|c|c|c|c} 
Delay (ns) & 20 & 70 & 130 & 190 & 310 & 330 \\
\hline Av. power (dB) & 0 & -3 & -10 & -18 & -26 & -32
\end{tabular}

We take $T_{h}=T_{h o}$ and perform $10^{4}$ Monte Carlo trials for each figure.

Figs. 3(a) to 3(c) show the RMS error for $\alpha=2,4$, and 8 . The number of taps in the ML estimator were 19, 15 and 13 for $\alpha=2,4$, and 8 respectively. We can see that estimator SP outperforms estimator ML significantly, and the improvement grows with the oversampling factor $\alpha$. In average, the improvement is $0.36 \mathrm{~dB}, 1.83 \mathrm{~dB}$, and $3.54 \mathrm{~dB}$ for oversampling factors $\alpha=2,4$, and 8 respectively. Estimator SP60 also outperforms estimator ML, though with a somewhat larger RMS error than estimator SP. 


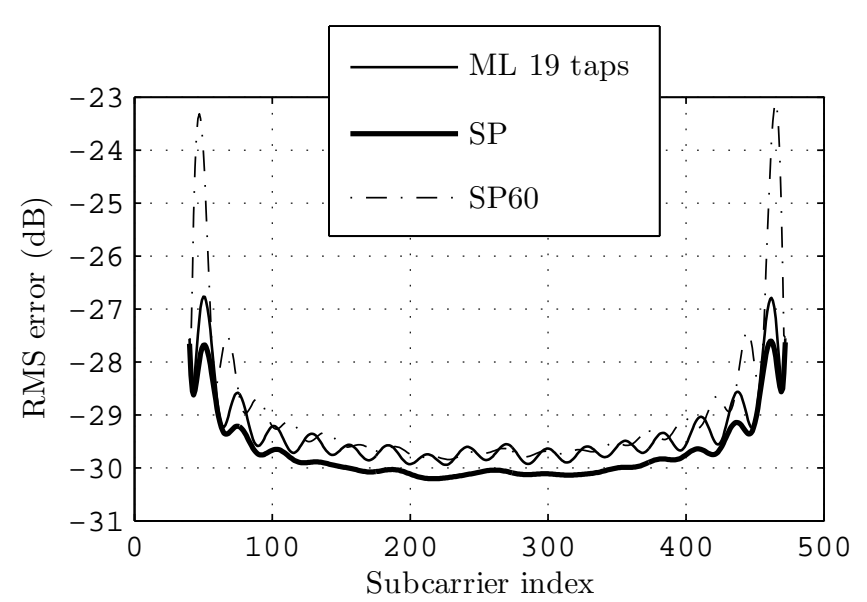

(a) $\alpha=2$.

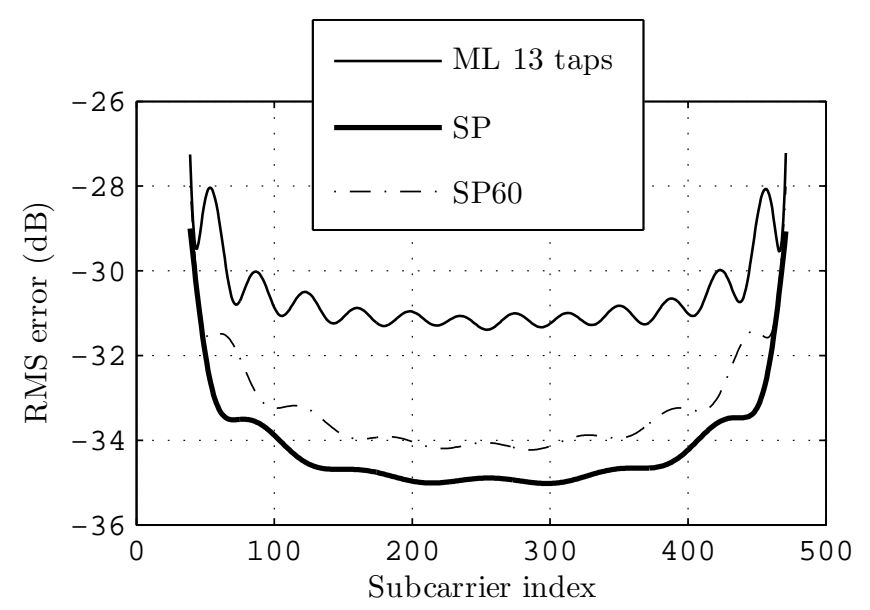

(c) $\alpha=8$.

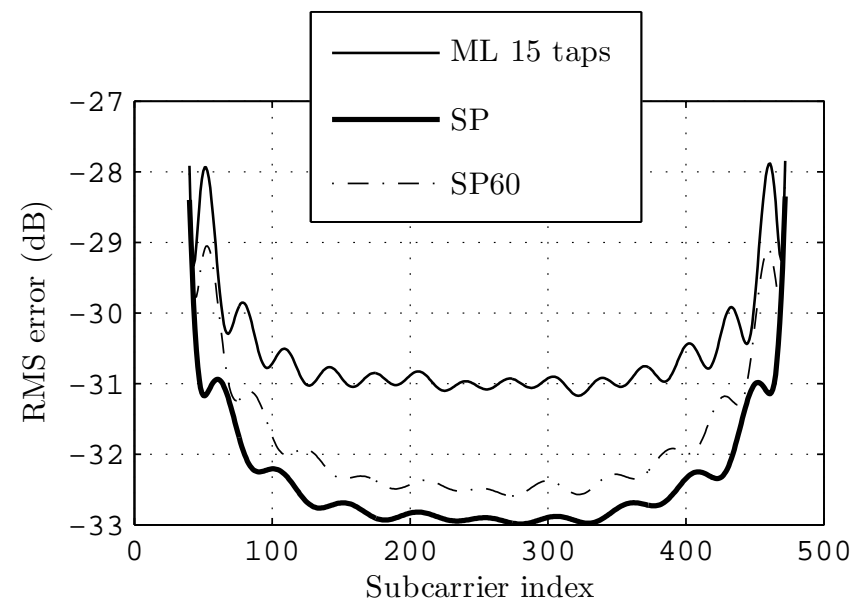

(b) $\alpha=4$.

Fig. 3. Bound and RMS error for the ML, SP, and SP60 estimators.

\section{Assessment for a nonuniform sampling scheme}

The previous examples were based on a uniform sampling scheme, in order to facilitate the comparisons with other results in the literature like [23, Sec. IV.C]. However, the SP and ML estimator are usable with nonuniform samples. A non-uniform distribution can be used to obtain a more uniform RMS error. Fig. 4 shows the repetition of Fig. 3(c) but for pilots placed in the OFDM frequency grid at positions close to the roots of a Chebyshev polynomial with proper scaling. Specifically, the pilots were placed at indices 40, 43, 48, 56, 67, 80, 95, 112, 131, 152, 173, 196, 220, 244, 268, 292, 316, 339, 360, 381, 400, $417,432,445,456,464,469$, and 472 . Note that, except at the frequency limits, the error distribution is more uniform and the SP estimator error is below $-31.5 \mathrm{~dB}$. 


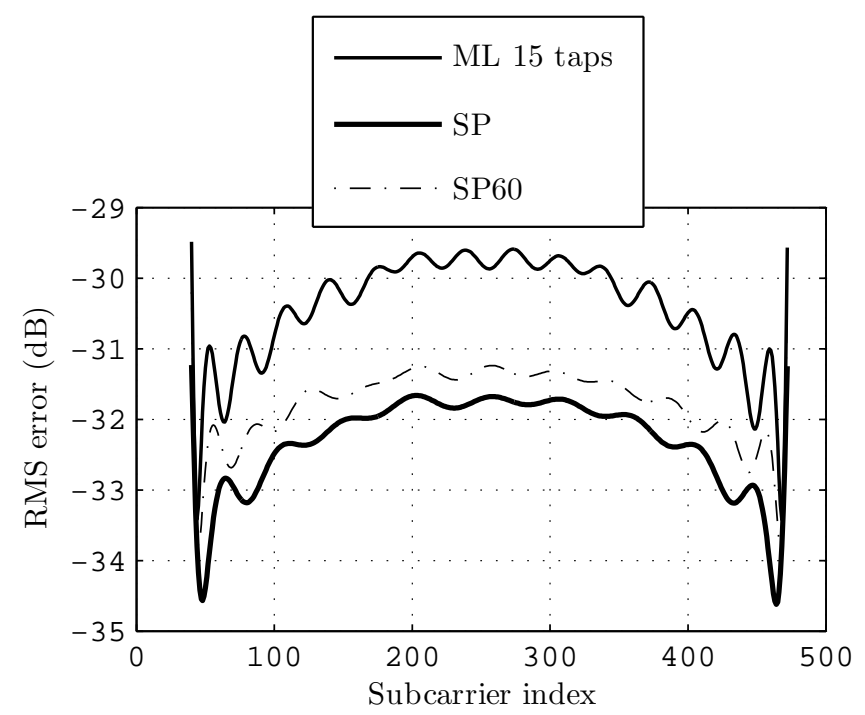

Fig. 4. RMS error for the Chebyshev sampling scheme with $\alpha=4$.

\section{CONCLUSIONS}

In this paper, we have discussed two approaches for designing an estimator for a static channel spectrum, that take as input nonuniform spectral samples. The first, called trigonometric approach, is the usual one in the literature, and is based on approximating the spectrum using a trigonometric polynomial. The second, called spectral approach, is the one proposed in this paper and introduces the delay spread as a new parameter in order to improve the estimation performance. Using this second approach, we have derived a linear estimator that approximately minimizes the RMS estimation error assuming a deterministic channel. We have shown in the numerical examples that the proposed method improves on the ML estimator based on the trigonometric approach in RMS error performance significantly. Besides, this improvement increases with the spectral oversampling factor.

\section{APPENDIX A}

A POSSIBLE PULSE $w(t)$

Consider the Kaiser-Bessel window

$$
w_{o}(t) \equiv \begin{cases}\frac{\mathrm{I}_{0}\left(\frac{\pi B_{w} T_{w}}{2} \sqrt{1-\left(\frac{2 t}{T_{w}}\right)^{2}}\right)}{\mathrm{I}_{0}\left(\pi B_{w} T_{w} / 2\right)} & \text { if }|t| \leq T_{w} / 2 \\ 0 & \text { otherwise, }\end{cases}
$$


where $\mathrm{I}_{0}$ is the modified Bessel function of the first kind and order zero, and $T_{w}$ and $B_{w}$ respectively denote the time- and frequency-domain widths of $w_{o}(t)$. The interval $\left[-T_{w} / 2, T_{w} / 2\right]$ contains all the energy of $w_{o}(t)$ in the time domain, whereas $\left[-B_{w} / 2, B_{w} / 2\right]$ contains most but not all of its energy in the frequency domain. Besides, the energy leakage outside $\left[-B_{w} / 2, B_{w} / 2\right]$ decreases exponentially with the product $B_{w} T_{w}$, and is negligible even for small values of $B_{w} T_{w}$, [7], [30], [31]. Assuming that this last leakage is negligible, we may construct from $w_{o}(t)$ a pulse $w(t)$ with small energy such that $W(f) \approx e^{-j \pi T_{w} f}$ if $f_{1} \leq f \leq f_{M}$. Let us write $w(t)$ and its time and frequency supports in the following way

$$
\begin{aligned}
& \text { Pulse: } w_{o}(t) \\
& \text { Supports: }\left[-T_{w} / 2, T_{w} / 2\right],\left[-B_{w} / 2, B_{w} / 2\right] .
\end{aligned}
$$

We may construct $w(t)$ in the following steps,

1) Construct a pulse with spectrum approximately equal to one in a band $\left[-B_{1} / 2, B_{1} / 2\right], B_{1}>0$, by convolving $W_{o}(f)$ with a rectangular pulse of width $B_{1}+B_{w}$,

$$
W_{o}(f) * \Pi\left(\frac{f}{B_{1}+B_{w}}\right) .
$$

Using the notation in (24), we obtain

$$
\begin{aligned}
& \text { Pulse: } w_{o}(t)\left(B_{1}+B_{w}\right) \operatorname{sinc}\left(\left(B_{1}+B_{w}\right) t\right) \\
& \text { Supports: }\left[-T_{w} / 2, T_{w} / 2\right],\left[-B_{w}-B_{1} / 2, B_{1} / 2+B_{w}\right] .
\end{aligned}
$$

2) Center the spectrum around a frequency $f_{c}$,

$$
\text { Pulse: } w_{o}(t)\left(B_{1}+B_{w}\right) \operatorname{sinc}\left(\left(B_{1}+B_{w}\right) t\right) e^{j 2 \pi f_{c} t},
$$$$
\text { Supports: }\left[-T_{w} / 2, T_{w} / 2\right] \text {, }
$$

$$
\left[f_{c}-B_{w}-B_{1} / 2, f_{c}+B_{1} / 2+B_{w}\right] \text {. }
$$

Now the spectrum is approximately equal to one in $\left[f_{c}-B_{1} / 2, f_{c}+B_{1} / 2\right]$.

3) Delay the last pulse by $T_{w} / 2$ to obtain a pulse with the desired time support $\left[0, T_{w}\right]$ and spectrum approximately equal to $e^{-j \pi T_{w} f}$ in $\left[f_{c}-B_{1} / 2, f_{c}+B_{1} / 2\right]$,

$$
\begin{aligned}
& \text { Pulse: } w_{o}\left(t-T_{w} / 2\right)\left(B_{1}+B_{w}\right) \\
& \quad \cdot \operatorname{sinc}\left(\left(B_{1}+B_{w}\right)\left(t-T_{w} / 2\right)\right) e^{j 2 \pi f_{c}\left(t-T_{w} / 2\right)},
\end{aligned}
$$

Supports: $\left[0, T_{w}\right]$,

$$
\left[f_{c}-B_{w}-B_{1} / 2, f_{c}+B_{1} / 2+B_{w}\right] .
$$

4) Set $B_{1}=f_{M}-f_{1}$ and $f_{c}=\left(f_{1}+f_{M}\right) / 2$ to obtain the final pulse

$$
\begin{aligned}
& \text { Pulse: } w_{o}\left(t-T_{w} / 2\right)\left(f_{M}-f_{1}+B_{w}\right) \\
& \quad \cdot \operatorname{sinc}\left(\left(f_{M}-f_{1}+B_{w}\right)\left(t-T_{w} / 2\right)\right) e^{j \pi\left(f_{1}+f_{M}\right)\left(t-T_{w} / 2\right)}, \\
& \text { Supports: }\left[0, T_{w}\right],\left[f_{1}-B_{w}, f_{M}+B_{w}\right] .
\end{aligned}
$$


whose spectrum is approximately equal to $e^{-j \pi T_{w} f}$ in $\left[f_{1}, f_{M}\right]$.

\section{REFERENCES}

[1] F. Marvasti, Ed., Nonuniform sampling, theory and practice, Kluwer academic/Plenum Publishers, 2001.

[2] J. J. Benedetto and P. J. S. G. Ferreira, Eds., Modern sampling theory, chapter 12, Birkhauser Boston, 2001.

[3] S. K. Sindhi and K.M.M. Prabhu, "Reconstruction of N-th order nonuniformly sampled bandlimited signals using digital filter banks," Digital Signal Processing, vol. 23, no. 6, pp. 1877-1886, 2013.

[4] K. M. Tsui and S. C. Chan, "A novel iterative structure for online calibration of M-channel time-interleaved ADCs," IEEE Transactions on Instrumentation and Measurement, vol. 63, no. 2, pp. 312-325, 2014.

[5] J. Selva, "Efficient sampling of band-limited signals from sine wave crossings," IEEE Transactions on Signal Processing, vol. 60, no. 1, pp. 503-508, Jan 2012.

[6] J. Selva, "Design of barycentric interpolators for uniform and nonuniform sampling grids," IEEE Transactions on Signal Processing, vol. 58, no. 3, pp. 1618 -1627, Mar 2010.

[7] J. Selva, "Functionally weighted Lagrange interpolation of band-limited signals from nonuniform samples," IEEE Transactions on Signal Processing, vol. 57, no. 1, pp. 168-181, Jan 2009.

[8] S. Tertinek and C. Vogel, "Reconstruction of nonuniformly sampled bandlimited signals using a differentiator-multiplier cascade," IEEE Transactions on Circuits and Systems - I: Regular papers, vol. 55, no. 8, pp. 2273-2286, Sep 2008.

[9] H. Johansson and P. Löwenborg, "Reconstruction of nonuniformly sampled bandlimited signals by means of time-varying discrete-time FIR filters,” EURASIP Journal on Applied Signal Processing, vol. 2006, pp. Pages 1-18, 2006.

[10] J. L. Yen, "On nonuniform sampling of bandwidth-limited signals," IRE Transactions on Circuit Theory, vol. CT-3, pp. 251-257, Dec 1956.

[11] J. Selva, "Optimal variable fractional delay filters in time-domain L-infinity norm," in International Conference on Acoustics Speech, and Signal Processing, ICASSP'-09, Apr 2009, pp. 3373-3376.

[12] K. Grochenig, "Irregular sampling, Toeplitz matrices, and the approximation of entire functions of exponential type," Mathematics of Computation, vol. 68, no. 226, pp. 749-765, 1999.

[13] H. G. Feichtinger, "Irregular sampling theorems and series expansions of band-limited functions," Journal of Mathematical Analysis and Applications, 1992.

[14] H. G. Feichtinger and K. Grochenig, "Theory and practice of irregular sampling," Wavelets: mathematics and applications, vol. 1994, pp. 305-363, 1994.

[15] H. G. Feichtinger, K. Gröchenig, and T. Strohmer, "Efficient numerical methods in non-unifrom sampling theory," Numerische Mathematik, vol. 69, pp. 423-440, 1995.

[16] K. Grochenig, "Reconstructions algorithms in irregular sampling," Mathematics of Computation, vol. 59, no. 199, pp. 181-194, Jul 1992.

[17] K. Grochenig and T. Strohmer, "Numerical and theoretical aspects of nonuniform sampling of band-limited images," in Nonuniform Sampling, pp. 283-324. Springer, 2001.

[18] T. Strohmer and J. Tanner, "Fast reconstruction methods for bandlimited functions from periodic nonuniform sampling," SIAM J. Numer. Anal., vol. 44, pp. 1073-1094, 2006.

[19] R. G. Lyons, Understanding Digital Signal Processing, Prentice Hall, 2001.

[20] M.K. Ozdemir and H. Arslan, "Channel estimation for wireless OFDM systems," IEEE Communications Surveys Tutorials, vol. 9, no. 2, pp. 18-48, 2007. 
[21] P. Fertl and G. Matz, "Channel estimation in wireless OFDM systems with irregular pilot distribution," IEEE Transactions on Signal Processing, vol. 58, no. 6, pp. 3180-3194, June 2010.

[22] L. Tong, B.M. Sadler, and M. Dong, "Pilot-assisted wireless transmissions: general model, design criteria, and signal processing," Signal Processing Magazine, IEEE, vol. 21, no. 6, pp. 12-25, 2004.

[23] M. Morelli and U. Mengali, "A comparison of pilot-aided channel estimation methods for OFDM systems," Signal Processing, IEEE Transactions on, vol. 49, no. 12, pp. 3065-3073, 2001.

[24] H. Meyr, M. Moeneclaey, and S. A. Fechtel, Digital Communications Receivers, vol. 2: Synchronization, Channel Estimation, and Signal Processing, Wiley-Interscience, 1997.

[25] M. Failli, “Digital land mobile radio communications COST 207,” Tech. Rep., European Commission, 1989.

[26] H. Schober, F. Jondral, R. Stirling-Gallacher, and Z. Wang, "Delay spread estimation for OFDM based mobile communication systems," in Proc. European Wireless Conf, 2002, pp. 625-628.

[27] W. Rudin, Real and complex analysis, Mathematics series. McGraw-Hill International Editions, 1987.

[28] K. E. Grue, "Optimal reconstruction of bandlimited bounded signals," IEEE Transactions on Information Theory, vol. IT-31, no. 5, pp. 594-601, Sept. 1985.

[29] Recommendation ITU-R M.1225, Guidelines for evaluation of radio transmission technologies for IMT-2000, 1997.

[30] J. J. Knab, "Interpolation of band-limited functions using the Approximate Prolate series," IEEE Transactions on Information Theory, vol. IT-25, no. 6, pp. 717-720, Nov 1979.

[31] J. Selva, "Regularized sampling of multiband signals," IEEE Transactions on Signal Processing, , no. 11, pp. 5624-5638, Nov 2010.

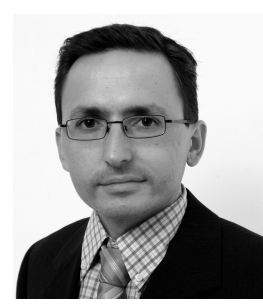

J. Selva received the M.Sc. degree in Communications Engineering from the Polytechnic University of Valencia (UPV), Valencia, Spain, in 1994, the M.Sc. degree in Mathematics from the UNED University, Madrid, Spain, in 2000, and the Ph.D. degree in Communications Engineering from the Polytechnic University of Catalonia (UPC), Barcelona, Spain, in 2004. During 1995, he was a grant holder at the Polytechnic University of Valencia. From 1996 to the end of 1998, he was a trainee of the Spanish Ministry of Education at the European Space Agency (ESA) sites in Frascati, Italy, and Noordwijk, The Netherlands. From 1998 to 2004, he was researcher with the German Aerospace Centre (DLR), Oberpfaffenhofen, Bayern, Germany. From 2004 to 2011, he was "Juan de la Cierva" and "Ramon y Cajal" Research Fellow at the University of Alicante, Spain. From 2012 to date he has been associate professor in this last university. His main research interests include interpolation for signal processing, sampling theory, navigation systems, synchonization circuits, array processing, and estimation theory. 